K. Nishioka

Nagoya Math. J.

Vol. 109 (1988), 63-67

\title{
A NOTE ON THE TRANSCENDENCY OF PAINLEVE'S FIRST TRANSCENDENT
}

\author{
KEIJI NISHIOKA
}

\section{$\S 1$. Introduction}

Here we shall prove that Painlevé's first transcendent, a solution of the equation $y^{\prime \prime}=6 y^{2}+x$, can not be described as any combination of solutions of first order algebraic differential equations and those of linear differential equations. This result gives an answer to the question whether the function is truely new or not.

To make the matter clear, we adopt the notions from differential algebra (refer to [2]). Let $K$ be an ordinary differential field of characteristic zero. In what follows we discuss everything in a fixed universal differential field extension of $K$. A differential field extension $L$ of $K$ will be said to be decomposable if there exists a differential field extension $M$ of $K$ such that $L$ is free from $M$ over $K$ and $L M$ is contained in the terminal $N_{n}$ of a finite chain of differential field extensions of

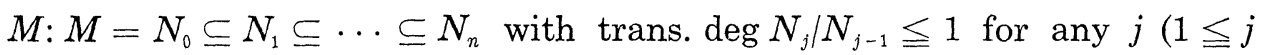
$\leqq n$ ). And we also say that a differential field extension $L$ of $K$ is $d e$ composable if there is a finite chain of differential field extensions of $K: K=L_{0} \subseteq L_{1} \subseteq \cdots \subseteq L_{m}=L$ such that for any $j(1 \leqq j \leqq m) L_{j}$ is a decomposable differential field extension of $L_{j-1}$ in the sense just defined above. For example a strongly normal extension of $K$ is decomposable. A differential field extension of $K$ which is generated with a solution of some linear differential equation over $K$ is decomposable (see $\S 3$ ). We shall prove the following:

THEOREM. Let a be a nonconstant element of $K$. Suppose that a solution $y$ of the equation $y^{\prime \prime}=6 y^{2}+a$ is contained in some decomposable differential field extension of $K$. Then $y$ is algebraic over $K$.

In particular, from this and the fact Painlevés first transcendent is

Received June 2, 1986. 
a transcendental function, it is not contained in any decomposable differential field extension of the algebraic closure $\overline{C(x)}$ of $C(x)$. Thus Liouville's claim [3] that Painlevé's first transcendent is expressible by a solution of some linear differential equation over $\overline{C(x)}$ is found to be false. Our proof of the theorem is simple and in some sense intimately related to the "irreducibility" of the function, though we do not use Drach's theory (for modern treatment upon Drach's theory, see [6], especially pp. 661-668).

\section{$\S 2$. Proof of the theorem}

The following fact is essential: Suppose that $L$ is a differential field extension of $K$ and a solution $y$ of the equation $y^{\prime \prime}=6 y^{2}+a$ satisfies trans. $\operatorname{deg} L\langle y\rangle \mid L \leqq 1$. Then $y$ is algebraic over $L$. In fact assume the converse. Then there is an irreducible polynomial $F$ in $L\left[Y, Y_{1}\right]$ such that $\partial F / \partial Y_{1}\left(y, y^{\prime}\right) \neq 0$ and $F\left(y, y^{\prime}\right)=0$. By the equalities for $y$ it is seen that the differential polynomial

$$
F^{D}+Y_{1} \partial F / \partial Y+\left(6 Y^{2}+a\right) \partial F / \partial Y_{1}
$$

vanishes at $y$, where $F^{D}$ denotes the polynomial obtained by differentiating the coefficients of $F$. Hence it is divisible by $F$ in the ring $L\left[Y, Y_{1}\right]$ because of the irreducibility of $F$. We define the weight $w(A)$ of a nonzero element $A$ of $L\left[Y, Y_{1}\right]$ as $w(A)=\max \left\{2 i+3 j ; a_{\imath j} \neq 0\right\}$, where $A=$ $\sum a_{i j} Y^{i} Y_{1}^{j}$. By $V_{m}$ we denote the linear subspace of $L\left[Y, Y_{1}\right]$ over $L$ which is generated with all monomial of weight $m$. Then we have the decomposition: $L\left[Y, Y_{1}\right]=\oplus_{0 \leqq m} V_{m}$. Define a derivation $X$ of $L\left[Y, Y_{1}\right]$ over $L$ by

$$
X=Y_{1} \partial / \partial Y+6 Y^{2} \partial / \partial Y_{1} .
$$

We find immediately $w(X A) \leqq w(A)+1$ for any $A$ and $X V_{m} \subseteq V_{m+1}$. Since

$$
w\left(F^{D}+X F+a \partial F / \partial Y_{1}\right) \leqq w(F)+1,
$$

it follows there is an element $b$ of $L$ with

$$
X F=b F-F^{D}-a \partial F / \partial Y_{1} .
$$

Let $p=u(F)$ and $F=\sum F_{m}$ be the decomposition, where $F_{m}=0$ for $m$ greater than $p$ and $F_{p} \neq 0$. Then

$$
X F_{m}=b F_{m+1}-F_{m+1}^{D}-a \partial F_{m+4} / \partial Y_{1} \quad(0 \leqq m) .
$$

From these equalities we determine $F_{m}$. Let $Z=Y_{1}^{2}-4 Y^{3}$. This poly- 
nomial is irreducible in $L\left[Y, Y_{1}\right]$ and satisfies $X Z=0$ and $w(Z)=6$. Then we have the following:

(*) Let $H$ be a nonzero element of $V_{m}$ and divisible strictly by $Z^{s}$. Set $H=\left(A Z+Y_{1} B+C\right) Z^{s}, A \in L\left[Y, Y_{1}\right], B$ and $C \in L[Y]$. Then

i) if $X H \neq 0, X H$ is divisible strictly by $Z^{s}$ and we have the expression: $X H=I Z^{s}$, where

$$
I=\left(X A+B_{Y}\right) Z+Y_{1} C_{Y}+2 Y^{2}\left(2 Y B_{Y}+3 B\right) ;
$$

ii) if $X H=0, m=6 s$.

In fact

$$
\begin{aligned}
X H & =\left[(X A) Z+Y_{1}^{2} B_{Y}+6 Y^{2} B+Y_{1} C_{Y}\right] Z^{s} \\
& =\left[\left(X A+B_{Y}\right) Z+Y_{1} C_{Y}+2 Y^{2}\left(2 Y B_{Y}+3 B\right)\right] Z^{s} .
\end{aligned}
$$

If $X H$ is divisible by $Z^{s+1}, Z$ divides the polynomial between above brackets. Hence $C$ lies in $L$ and $2 Y B_{Y}+3 B=0$, which implies $B=0$. By the assumption on $H$ we see $C \neq 0$ and so $H=C Z^{s}$, particularly $X H=0$ and $m=6 s$. This completes the proof.

By the equality $(p)$ we have $X F_{p}=0$ and because of $\left(^{*}\right)$ we have the representation

$$
F_{p}=c Z^{q}, \quad c \in L, \quad p=6 q .
$$

By the equality $(p-1), X F_{p-1}=\left(b c-c^{\prime}\right) Z^{q}$. According to $\left(^{*}\right)$ and that $p-1$ is not divisible by 6 , we have $F_{p-1}=0$. Hence $X F_{p-2}=b F_{p-1}-$ $F_{D}^{p-1}=0$ by the equality $(p-2)$, and $F_{p-2}=0$ by $\left(^{*}\right)$ and because $p-2$ is not divisible by 6 . Similarly we see $F_{p-3}=0$. By the equality $(p-4)$ we have

$$
X F_{p-4}=-a F_{p, Y_{1}}=-2 a c q Y_{1} Z^{q-1} .
$$

By $(*)$ if we use the same notations appeared there

$$
C_{Y}=-2 a c q, \quad 2 Y B_{Y}+3 B=0 .
$$

Hence $B=0, C=-2 a c q Y, A=0$. Consequently

$$
F_{p-4}=-2 a c q Y Z^{q-1} \text {. }
$$

By the equality $(p-5)$,

$$
X F_{p-5}=b F_{p-4}-F_{p-4}^{D}-a F_{p-1, Y_{1}}=2 a^{\prime} c q Y Z^{q-1} .
$$

This implies $a^{\prime}=0$ by $(*)$ and our assertion has been proved. 
Let us return to the proof of our theorem. Let $R$ and $S$ be two differential field extensions of $K$. Suppose that $R$ contains a solution $y$ of the equation $y^{\prime \prime}=6 y^{2}+a$, being free from $S$ over $K$, and there is a chain of differential field extensions of $S: S=T_{0} \subseteq T_{1} \subseteq \cdots \subseteq T_{n}$ with trans. deg $T_{j} / T_{3-1} \leqq 1$ for any $j(1 \leqq j \leqq n)$ and $T_{n} \supseteq R S$. Since $y$ lies in $R S$ therefore in $T_{n}$, because of the fact just proved above, it follows that $y$ is algebraic over $S$. Since $R$ and $S$ are free over $K, y$ is algebraic over $K$. Repeating the same argument, we may complete the proof of the theorem.

\section{§3. Rational dependence on arbitrary constants}

To explain examples mentioned in Section 1, we shall introduce a notion. We say that a differential field extension $L$ of $K$ depends rationally on arbitrary constants if there exists a differential field extension $M$ of $K$ such that $L$ and $M$ are free over $K$ and $L M=M C_{L M}$, where $C_{L M}$ denotes the field of constants of $L M$. Such extensions are decomposable, if the field $L$ is finitely generated over $K$. In fact $C_{L M}$ is finitely generated over $C_{M}$ by Corollary 1 in [2, p. 113], so that we have $C_{L M}=C_{M}\left(c_{1}, c_{2}, \cdots, c_{n}\right)$. Let $N_{j}$ be $M\left(c_{1}, c_{2}, \cdots, c_{j}\right)$. They satisfy the required conditions and $L$ is decomposable.

Let $L$ be a strongly normal extension of $K$. By the definition of strongly normal extension there is a differential field extension $M$ of $K$ such that $M$ is differentially isomorphic to $L$ over $K, L$ and $M$ are free over $K$ and $L M=M C_{L M}$ (cf. Proposition 1 in [1]). Thus $L$ depends rationally on arbitrary constants.

Let $y$ be a solution of the following linear differential equation over $K$ :

$$
y^{(n)}+p_{1} y^{(n-1)}+\cdots+p_{n} y=q .
$$

We show the differential field extension $K\langle y\rangle$ of $K$ depends rationally on arbitrary constants. We may assume that $K$ is algebraically closed. There exists a differential field extension $M$ of $K$ such that $M$ is free from $K\langle y\rangle$ over $K$, contains a solution $z$ of the same linear differential equation as above and a fundamental system $y_{1}, \cdots, y_{n}$ of solutions of the equation

$$
y^{(n)}+p_{1} y^{(n-1)}+\cdots+p_{n} y=0
$$


(see p. 746 in [4]). Then in $L M$,

$$
y=z+\sum c_{j} y_{j}, \quad c_{j} \in C_{L M} .
$$

This shows that our assertion is true.

For detail discussion on the relations between strongly normal extensions and differential field extensions depending rationally on arbitrary constants one may consult [7], where Painlevé's several assertions stated in his Stockholm lessons are restated in the language of algebraic geometry and proved rigorously.

\section{REFERENCES}

[1] A. Bialynicki-Birula, On Galois theory of fields with operators, Amer. J. Math., 84 (1962), 89-109.

[2] E. R. Kolchin, Differential Algebra and Algebraic Groups, Academic Press, New York, 1973.

[ 3 ] R. Liouville, Sur les équations différentielles du second order à points critiques fixes, Oevres de P. Painlevé, vol. 3 (1975), pp. 81-83.

[4] K. Nishioka, A class of transcendental functions containing elementary and elliptic ones, Osaka J. Math., 22 (1985), 743-753.

[5] K. Okamoto, Introduction to the Painlevé Equations (in Japanese), Sophia Kokyuroku in Math., vol. 19, 1985.

[6] J. F. Pommaret, Differential Galois theory, Gordon and Breach, 1983.

[7] H. Umemura, Birational automorphism groups and differential equations, to appear.

Takabatake-cho 184-632

Nara, 630, Japan 